\title{
Automatisation des comparateurs de courants électriques alternatifs pour la mesure des fortes intensités au LNE
}

\author{
Automation of the electric AC current \\ comparators for high current measurements \\ at LNE
}

\author{
Mohamed AGAZAR et Denis BÉLIÈRES
}

Laboratoire national de métrologie et d'essais (LNE), 29 Avenue Roger Hennequin, 78197 Trappes Cedex, France, mohamed.agazar@lne.fr.

\section{Résumé}

Deux comparateurs de courants à annulation de flux magnétiques ont été conçus dans les années 1970 au LCIE (Laboratoire Central des Industries Electriques) pour servir de référence métrologique pour l'étalonnage des transformateurs de courant de fortes intensités jusqu'à $50 \mathrm{kA}$ aux fréquences industrielles $50 \mathrm{~Hz}, 60 \mathrm{~Hz}$ et $400 \mathrm{~Hz}$. Les dispositifs électroniques auxiliaires ont également été conçus, il s'agit d'un dispositif de charge fictive et d'un dispositif d'injection de courant d'erreur capable de fournir avec une grande exactitude les composantes en phase et en quadrature de l'erreur du transformateur de courant à étalonner. Cet article présente les travaux effectués au LNE pour redéfinir entièrement ces deux dispositifs en abandonnant le système à asservissement direct de l'ancien équipement et en le remplaçant par un système à asservissement par logiciel offrant la possibilité d'améliorer la stabilité et l'incertitude de mesure et de contrôler l'ensemble à distance via une connexion USB et donc d'automatiser complètement les mesures.

MOTS CLÉS : COMPARATEUR DE COURANTS, DISPOSITIF D'INJECTION, CHARGE FICTIVE, TRANSFORMATEUR DE COURANT, ERREUR DE RAPPORT, DÉPHASAGE.

\section{Abstract}

Two compensated current comparators with magnetic flux cancelling have been made in the 1970s by the LCIE (Laboratoire Central des Industries Electriques) as a reference for high current transformers calibration for currents up to $50 \mathrm{kA}$ at industrial frequencies $50 \mathrm{~Hz}$, $60 \mathrm{~Hz}$ and $400 \mathrm{~Hz}$. They are associated with manually operated electronic equipments: an adjustable phantom burden and a ratio error set capable to provide with high accuracy the quadrature and the in-phase components of the current transformer errors. This article presents the work that has been done to entirely redefine theses devices by abandoning the idea of a direct servo system of the old equipment and replacing it by a software servo that offers high calibration stability, better incertainties and fully controllable by a remote computer via an USB connection, allowing full automation of the procedure.

KEYWORDS: COMPENSATED CURRENT COMPARATOR, PHANTOM BURDEN, RATIO ERROR SET, CURRENT TRANSFORMER, RATIO ERROR, PHASE-SHIFT.

\section{Introduction}

Deux comparateurs de courants à rapports multiples ont été conçus au LCIE pour servir de référence métrologique notamment pour l'étalonnage des transformateurs de courant et des shunts aux fréquences industrielles de $50 \mathrm{~Hz}, 60 \mathrm{~Hz}$ et de $400 \mathrm{~Hz}$. Le premier dénommé « petit comparateur » pour les rapports de 1 à 200 et le deuxième dénommé «grand comparateur» pour les rapports de 5 à 5 000. Pour un courant secondaire de $5 \mathrm{~A}$, les courants nominaux s'étendent jusqu'à $1 \mathrm{kA}$ pour le petit comparateur et jusqu'à $25 \mathrm{kA}$ pour le grand comparateur. L'association en cascade des deux comparateurs peut permettre d'étendre la gamme des rapports à 10000 en vue de traiter des courants s'étendant jusqu'à 50 kA.

Pour faciliter l'emploi de ces comparateurs, deux dispositifs électroniques ont également été conçus, il s'agit d'un dispositif de charge fictive et d'un dispositif d'injection de courant d'erreur capable d'afficher avec une grande exactitude les composantes en phase et en quadrature du courant d'erreur du transformateur à étalonner. À l'époque, la mise en œuvre de ces équipements 
électroniques a été très délicate en raison de la faible performance des composants utilisés, et de la volonté de réaliser un système asservi à lecture directe pouvant s'adapter à tous les rapports du comparateur. Cela suppose un bouclage du système électronique en contre-réaction au travers des enroulements du comparateur et des enroulements du transformateur à étalonner alors qu'ils peuvent en être éloignés de plusieurs mètres voire dizaines de mètres dans le cas d'un étalonnage sur un site industriel. L'exactitude de mesure demandée nécessite l'usage d'amplificateurs opérationnels à large bande passante (idéalement de plusieurs mégahertz pour des mesures à $400 \mathrm{~Hz}$ ), et la stabilité de tels amplificateurs est incertaine lorsqu'ils sont chargés par des réactances importantes, en particulier par la capacité des câbles blindés. Ces équipements électroniques ont été entièrement redéfinis en abandonnant l'idée d'un système asservi à lecture directe en le remplaçant par un système à asservissement par logiciel. Ils sont dorénavant entièrement contrôlables à distance par un ordinateur via une connexion USB, ce qui permet une automatisation complète : il n'y a plus aucune commande manuelle, que ce soit des commutateurs ou des potentiomètres, elles ont toutes été remplacées par des circuits électroniques.

\section{2. État de l'art}

Très peu de laboratoires nationaux emploient un comparateur de courants à annulation de flux magnétiques pour l'étalonnage des transformateurs de courant alternatif de fortes valeurs d'intensité. Cela nécessite une construction rigoureuse et une maitrise parfaite des erreurs magnétiques et capacitives. La plupart utilisent des comparateurs de courants classiques (semblables dans le principe à ceux utilisés pour l'étalonnages des condensateurs hautes tensions). L'étalonnage est fait le plus souvent par comparaison à un transformateur étalon et les incertitudes sont au mieux égales à $50 \mu \mathrm{A} / \mathrm{A}$ pour l'erreur de rapport et $50 \mu \mathrm{rad}$ pour le déphasage.

\section{Principe de fonctionnement}

\subsection{Définitions}

Le transformateur de courant est un convertisseur courant-courant capable de mesurer des intensités élevées (plusieurs kilo-ampères) en réduisant la valeur du courant aux valeurs normalisées de $1 \mathrm{~A}$ ou $5 \mathrm{~A}$. Le courant appliqué à son entrée traverse l'enroulement primaire; il est appelé le courant primaire. Le courant délivré en sa sortie traverse l'enroulement secondaire; il est appelé le courant secondaire.

Le rapport de transformation, indiqué dans la plaque signalétique du transformateur, est le rapport entre le courant primaire et le courant secondaire.

L'erreur de rapport est l'erreur que le transformateur introduit dans la mesure/transformation d'un courant. On cherche à déterminer l'erreur relative de ce rapport.

Le déphasage est la différence de phase entre les courants primaire et secondaire, le déphasage est considéré

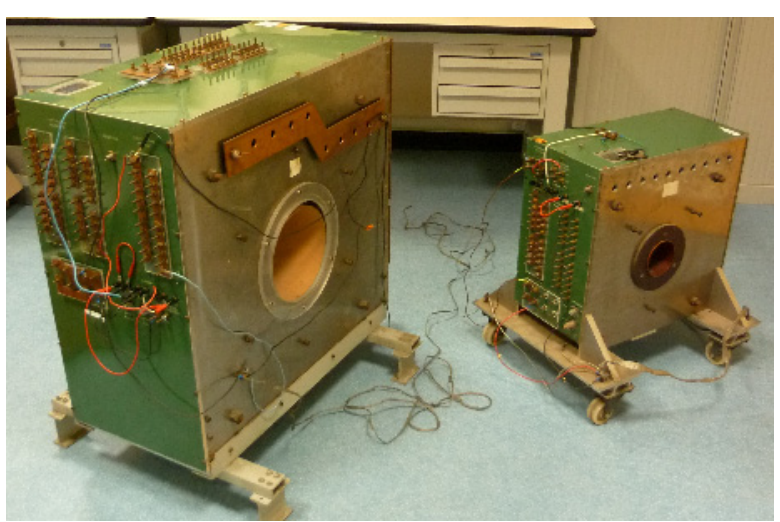

Fig. 1. - Le petit et le grand comparateurs de courants du LNE.

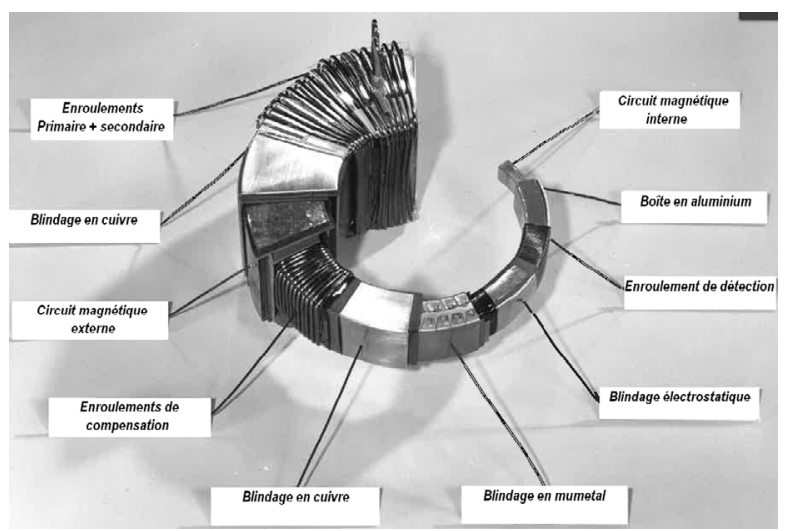

Fig. 2. - Composition d'un comparateur de courants.

positif si le courant secondaire est en avance sur le courant primaire.

Le circuit secondaire est le circuit extérieur alimenté par l'enroulement secondaire d'un transformateur.

La charge secondaire est l'impédance, à la fréquence nominale, du circuit secondaire. Elle est généralement caractérisée par la puissance apparente absorbée pour un facteur de puissance donné.

Le comparateur de courants à annulation de flux magnétiques est un transformateur de courant amélioré, il est considéré parfait grâce aux différents enroulements de compensation qu'il comporte (voir § 3.2). Il est doté de deux circuits magnétiques, le premier joue le même rôle que celui d'un transformateur de courant, le second sert à détecter un résidus de flux provenant d'un autre transformateur lorsque l'enroulement secondaire de ce dernier est connecté en série à l'enroulement secondaire du comparateur. Evaluer la quantité de ce flux revient donc à évaluer l'erreur du transformateur de courant.

\subsection{Composition du comparateur de courants}

La figure 1 présente la structure externe des comparateurs de courant du LNE. Leur composition interne est présenté dans la figure 2. Le principe de leur construction est détaillé dans l'article [1]. 
Pour rappel, chaque comparateur est composé essentiellement des éléments suivants :

- un circuit magnétique intérieur généralement en permimphy;

- un enroulement de détection entourant le circuit magnétique intérieur. Son rôle est de détecter la condition d'annulation de la tension induite apparaissant entre ses bornes ;

- un écran magnétique en mumétal entourant l'enroulement de détection et le circuit magnétique intérieur;

- un enroulement de compensation secondaire entourant tous les éléments précédents. Il permet de compenser l'effet des capacités parasites de l'enroulement secondaire. Il doit avoir le même nombre de spires et le même couplage que l'enroulement secondaire ;

- un enroulement de compensation primaire entourant tous les éléments précédents. Il permet de compenser l'effet des capacités parasites de l'enroulement primaire. Il doit avoir le même nombre de spires et le même couplage que l'enroulement primaire. Il permet éventuellement de compenser l'effet des capacités parasites de la source de courant primaire;

- un enroulement de compensation complémentaire. Il permet éventuellement de compenser les influences perturbatrices des capacités parasites de l'enroulement secondaire;

- un circuit magnétique extérieur entourant tous les éléments précédents;

- un enroulement secondaire entourant tous les éléments précédents ;

- un enroulement primaire entourant tous les éléments précédents.

En outre des écrans électrostatiques et des blindages permettent d'éliminer les couplages capacitifs directs entre les enroulements. Ils sont disposés comme suit :

- à l'intérieur et à l'extérieur de l'enroulement de détection;

- à l'intérieur de l'enroulement de compensation secondaire;

- à l'extérieur de l'enroulement de compensation complémentaire;

- à l'extérieure et à l'extérieur de l'enroulement secondaire;

- à l'intérieur et à l'extérieur de l'enroulement primaire.

\subsection{Etalonnage d'un transformateur de courant à l'aide d'un comparateur de courants}

Le montage utilisé $[2,3]$ pour étalonner un transformateur de courant à l'aide d'un comparateur de courants est présenté dans les figures 3 et 4 . Le comparateur de

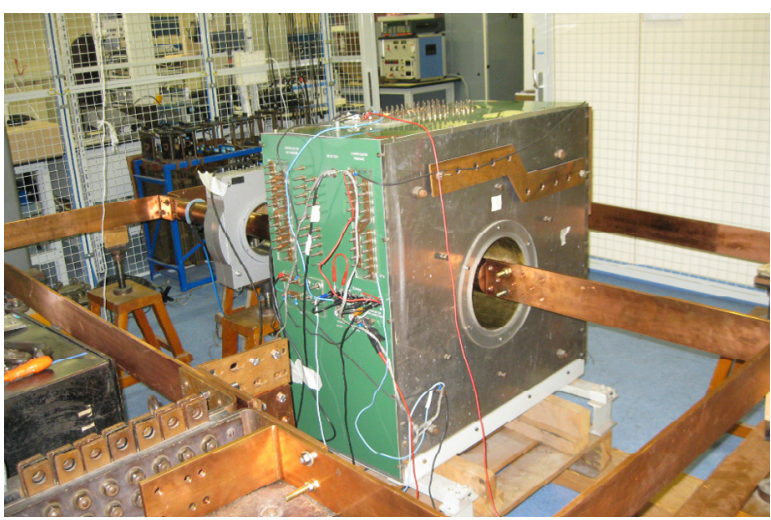

Fig. 3. - Le grand comparateur de courants du LNE dans un montage d'étalonnage d'un transformateur de courant.

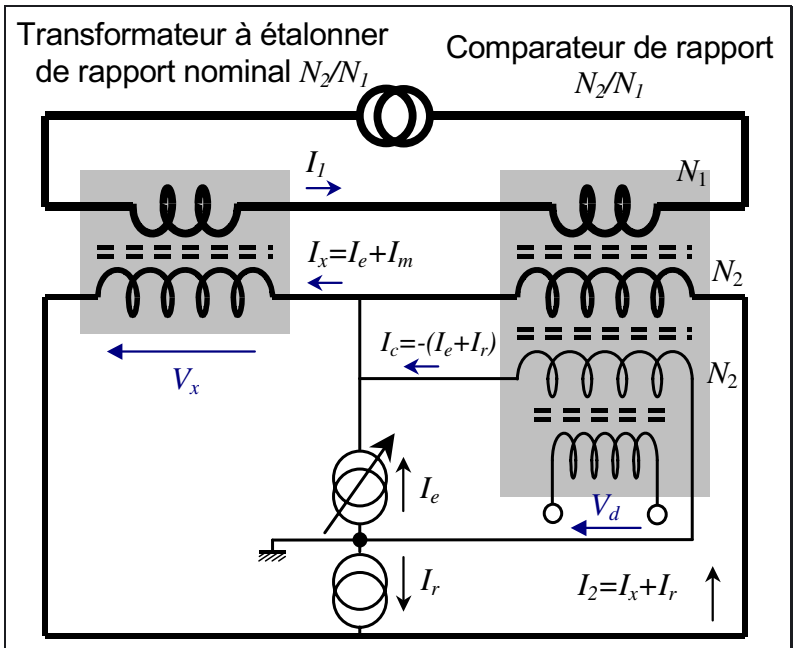

Fig. 4. - Principe d'étalonnage d'un transformateur de courant à l'aide d'un comparateur de courants.

courants et le transformateur de courant sont de même rapport (rapport entre le nombre de spires de l'enroulement secondaire et le nombre de spires de l'enroulement primaire) et sont traversés par le même courant (intensité) primaire $I_{1}$. L'enroulement secondaire est connecté à la terre par l'intermédiaire de l'enroulement de compensation secondaire. Dans ces conditions, un déséquilibre des courants primaire et secondaire produit une excitation des circuits magnétiques, ce qui permet de fournir de l'énergie à une charge disposée dans le circuit secondaire.

Dans la figure 4, les notations suivantes sont utilisées :

$I_{2}$ : composante fondamentale de l'intensité du courant circulant dans l'enroulement secondaire du comparateur de courants.

$I_{x}$ : composante fondamentale de l'intensité du courant circulant dans l'enroulement secondaire du transformateur à étalonner.

$I_{C}$ : composante fondamentale de l'intensité du courant circulant dans l'enroulement de compensation secondaire. 
$I_{e}$ : composante fondamentale de l'intensité du courant d'erreur du transformateur à étalonner.

Avec les conventions de signe de la figure 4 , on peut écrire :

$$
I_{x}=I_{2}+I_{c}+I_{e} .
$$

L'intensité du courant métrologique $I_{m}$, c'est-à-dire celle qui correspond à l'absence d'erreur s'écrit :

$$
I_{m}=I_{2}+I_{c} .
$$

Pour un transformateur de courant sans erreur :

$$
N_{1} I_{1}=N_{2} I_{x}=N_{2}\left(I_{2}+I_{c}\right) .
$$

Finalement, en présence d'erreur :

$$
N_{1} I_{1}=N_{2}\left(I_{x}-I_{e}\right) .
$$

$I_{e}$ exprime directement les deux composantes en phase et en quadrature de l'erreur du transformateur à étalonner.

Pour évaluer ces deux composantes, un générateur d'appoint est utilisé pour annuler la tension $V_{d}$ aux bornes de l'enroulement de détection en injectant dans l'enroulement de compensation secondaire le courant (ou intensité) $I_{e}$. Le dispositif d'injection de courant d'erreur assure le rôle de cette source d'appoint.

Le courant (ou intensité) $I_{r}$, fourni par une autre source d'appoint, sert à magnétiser le comparateur de courants et il est réglé de façon à donner la valeur complexe désirée à la charge du transformateur à étalonner, définie par le quotient de la tension à ses bornes secondaires $V_{x}$ par l'intensité de son courant secondaire $I_{x}$, il traverse à flux soustractif l'enroulement secondaire et l'enroulement de compensation secondaire et il n'a aucun effet métrologique sur le flux aux bornes de l'enroulement de détection. Le dispositif de charge fictive assure le rôle de cette source d'appoint (voir [4] pour plus de détails).

\subsection{Le système d'injection du courant d'erreur}

Le dispositif d'injection de courant d'erreur, dont le principe est présenté dans les figures 5, 6 et 7, a pour but d'injecter dans l'enroulement de compensation secondaire le courant (ou intensité) $I_{e}$ proportionnel à $I_{x}$ afin trouver la condition d'équilibre aux bornes de l'enroulement de détection $V_{d}=0$, contrôlée au moyen d'un détecteur synchrone. Ce courant présente directement les deux composantes en phase et en quadrature de l'erreur du transformateur.

Trois systèmes d'injection de courant sont possibles : à lecture directe, à lecture indirecte et système asservi.

\subsubsection{Le système d'injection à lecture directe}

Dans ce système (fig. 5), l'intensité $I_{e}$ est proportionnelle à $I_{x}$ à chaque instant, le gain complexe $k=a+j b$

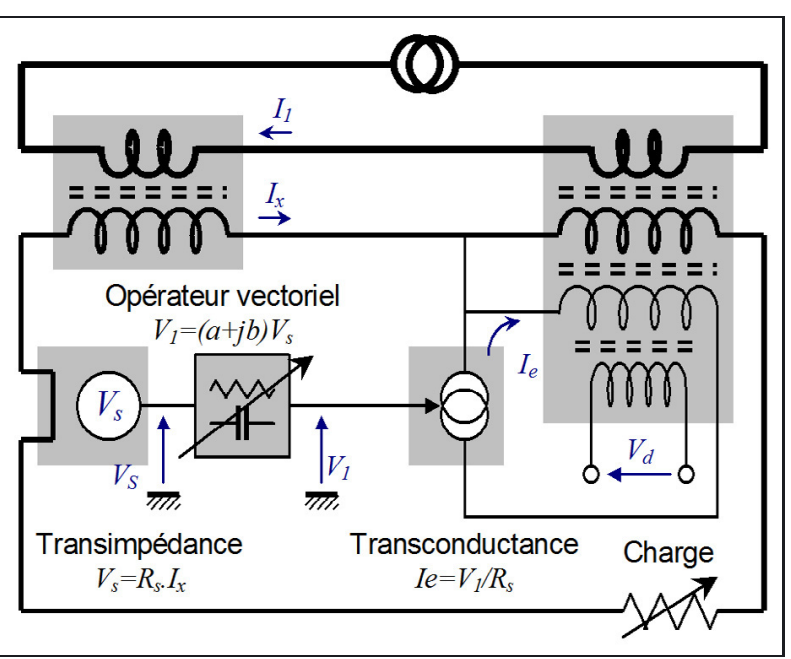

Fig. 5. - Principe du système d'injection à lecture directe.

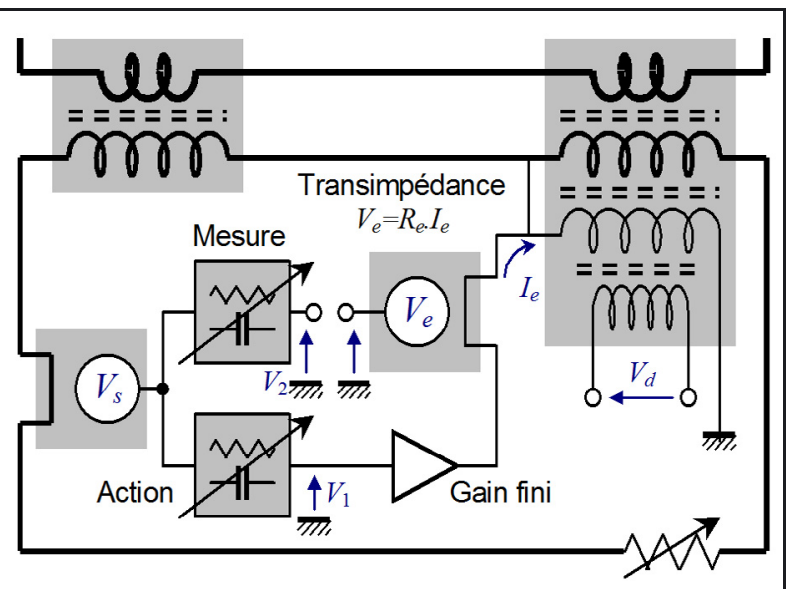

Fig. 6. - Principe du système d'injection à lecture indirecte.

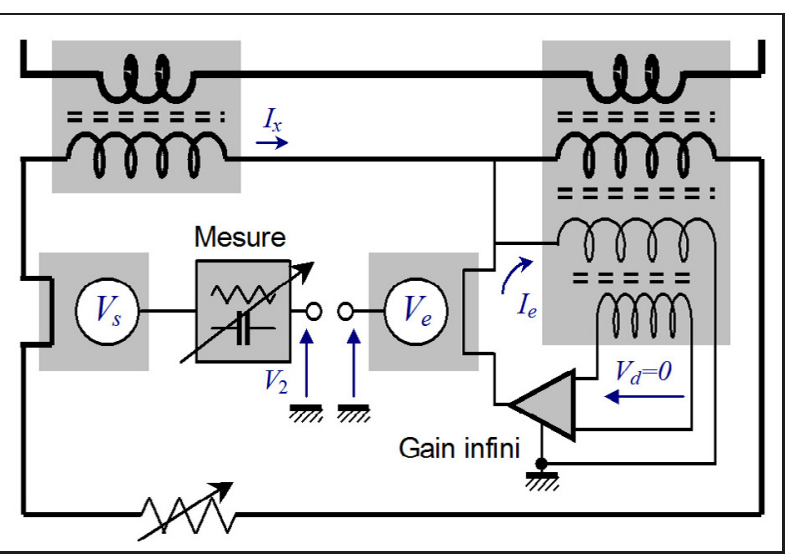

Fig. 7. - Principe du système d'injection asservi.

est réglable pour annuler la tension $V_{d}$. Ce gain complexe est, à un facteur d'échelle près, la transmittance d'un opérateur vectoriel fait d'un réseau commutable de résistances et de condensateurs connus. Cette transmittance est nécessairement dépendante de la fréquence, c'est-àdire que le courant délivré aura un contenu harmonique lié à de celui de $I_{x}$, mais pas ajustable indépendamment. 
Le système n'est donc pas utilisable pour mesurer un résidu harmonique.

\subsubsection{Le système d'injection à lecture indirecte}

La réalisation d'une véritable source de courant commandée présentant une transconductance parfaitement connue est difficile à concevoir surtout si on veut s'assurer qu'il n'y ait absolument aucune composante continue. Mais on peut se contenter d'une source d'impédance quelconque (soit une source de tension imparfaite) à condition de mesurer le courant produit (fig. 6). Dans ce système, un premier opérateur vectoriel dit «d'action » est réglé pour annuler la tension aux bornes de l'enroulement de détection contrôlée au moyen d'un détecteur synchrone.

Pour donner à l'appareil sa fonction métrologique d'affichage direct de la valeur relative du courant d'erreur $I_{e}$, ce dernier est mesuré via un shunt inséré en sortie du dispositif, qui constitue son impédance de sortie, et ensuite comparé au signal de référence issu d'un deuxième opérateur vectoriel dit «de mesure ». À l'équilibre du pont, contrôlé au moyen d'un détecteur synchrone, le dispositif d'injection permet d'afficher les composantes en phase et en quadrature du courant d'erreur $I_{e}$ avec une résolution de l'ordre de $5 \times 10^{-8}$ en valeur relative de $I_{x}$.

\subsubsection{Le système d'injection asservi}

Une variante du montage consiste en la fourniture de ce courant $I_{e}$ par un amplificateur opérationnel à l'entrée duquel est appliquée la tension détectée (fig. 7).

Dans toute la bande passante de l'asservissement ainsi réalisé, la tension détectée en entrée est maintenue à zéro, c'est-à-dire que $I_{e}$ est bien le courant d'erreur avec toutes ses composantes harmoniques. Mais, bien que ce courant circule dans l'enroulement de compensation avec sa valeur exacte sans besoin de réglages, il est tout de même inconnu et doit être mesuré. Pour cela, on procède comme précédemment en utilisant le seul opérateur vectoriel de mesure. Le comportement de cet opérateur vis-à-vis des harmoniques est sans importance, car après cette première mesure, c'est une simple analyse spectrale de la tension $V_{e}$ image de l'intensité $I_{e}$ qui peut en donner toutes les autres composantes voulues, rapportées au fondamental connu. Ce système d'asservissement est séduisant mais le bouclage de l'amplificateur avec le comparateur se fait au travers de câbles de grande longueur (quelques mètres) et des enroulements du comparateur qui sont faiblement couplés et présentent des capacités parasites importantes. Les déphasages provoqués dans cette contre-réaction sont très néfastes à la stabilité de la boucle, et en raison du grand nombre de configurations du comparateur il est impossible de les compenser et d'obtenir un asservissement inconditionnellement stable.

Par sa simplicité de conception, nous avons exploité le système d'asservissement à lecture indirecte.

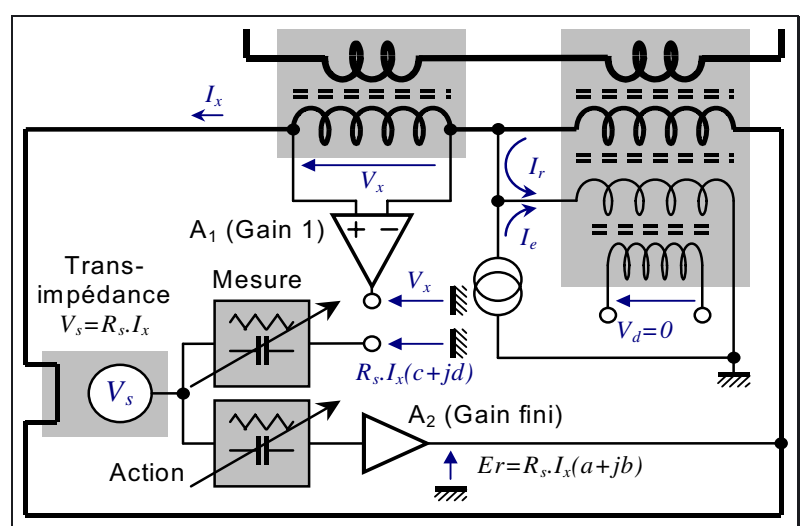

Fig. 8. - Système de charge fictive réglable.

Dans les figures 5, 6 et 7, le rôle de la transimpédance (décrite ultérieurement) est de fournir le signal de référence $V_{s}$ proportionnel à $I_{x}$ à partir duquel les deux vecteurs d'action et de mesure sont conçus.

\subsection{Le dispositif de charge fictive}

Le dispositif de charge fictive a pour but de fournir le courant (ou intensité) $I_{r}$ qui permet de magnétiser le comparateur de courants afin de fournir de l'énergie à une charge disposée dans son circuit secondaire. Le courant $I_{r}$ est en fait égal à la différence des courants magnétisants du comparateur et du transformateur. La charge fictive peut donc soit absorbé soit fournir de l'énergie $[4,5]$. Elle permet d'asservir avec exactitude et avec une grande résolution la composante complexe $Z=V_{x} / I_{x}$ qui caractérise la charge du transformateur à étalonner, c'est-à-dire, asservir la tension secondaire complexe $V_{x}$ du transformateur à étalonner et la maintenir à une valeur donnée proportionnelle au courant $I_{x}$. Cette tension est contrôlée en appliquant une tension $E_{r}$ entre les enroulements secondaire et de compensation du comparateur connectés en série et à flux soustractif donc le courant $I_{r}$ qu'elle fait circuler est sans effet sur la tension détectée $V_{d}$.

Deux systèmes de charge fictive sont possibles : charge réglable et charge asservie.

\subsubsection{La charge fictive réglable}

Dans ce système, la tension $V_{x}$ apparaissant aux bornes du secondaire du transformateur à étalonner (fig. 8) est réglée en appliquant une tension $E_{r}$ entre les enroulements secondaire et de compensation secondaire du comparateur connectés en série. Le courant $I_{r}$, généré par $E_{r}$, est sans effet sur la tension détectée $V_{d}$. On peut lier cette tension $E_{r}$ au courant secondaire $I_{x}$ en utilisant un nouvel opérateur vectoriel dit d'action, dont on règle la transmittance pour que la tension $V_{x}$ soit celle que l'on désire.

Pour cela, on la compare à la tension $R_{s} I_{x}(c+j d)$ sortant d'un autre opérateur vectoriel de mesure, et cette comparaison s'effectue en mettant ces deux tensions en 


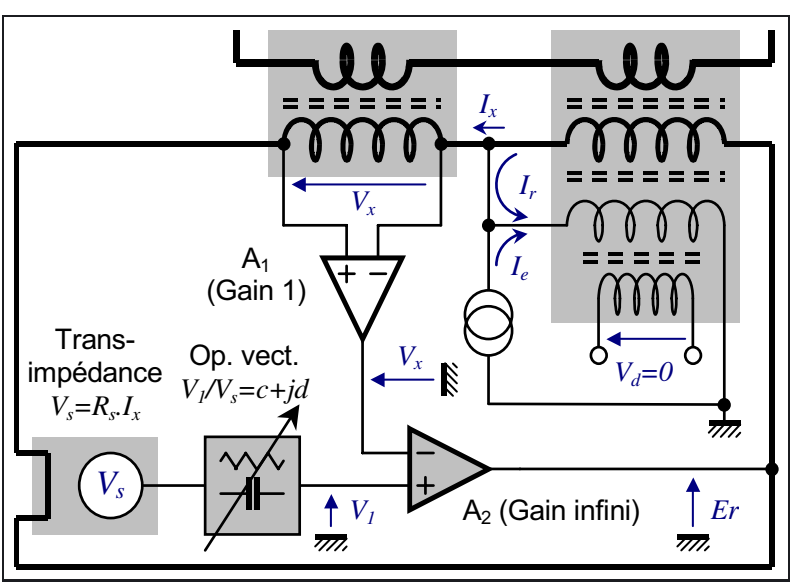

Fig. 9. - Système de charge fictive asservie.

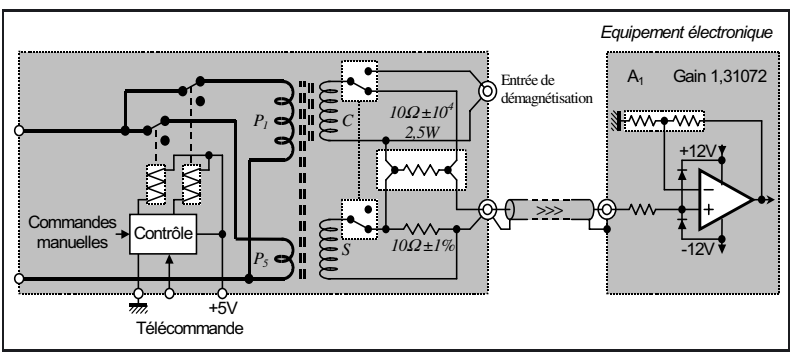

Fig. 10. - Le circuit à transimpédance.

opposition à l'entrée d'un détecteur synchrone. L'opérateur d'action est suivi d'un amplificateur $\mathrm{A}_{2}$ qui dans certains cas doit pouvoir fournir ou absorber une puissance importante. Le circuit à transimpédance de mesure du courant $I_{x}$ peut bien entendu être celui qui sert déjà au dispositif de mesure du courant d'erreur.

\subsubsection{La charge fictive asservie}

On peut fermer la boucle en asservissant la tension $V_{x}$ aux bornes du circuit secondaire sur la consigne $V_{1}=R_{s} I_{x}(c+j d)$ fournie par l'opérateur vectoriel de mesure (fig. 9). L'amplificateur différentiel $\mathrm{A}_{1}$ assure le retour de la contre-réaction de l'amplificateur opérationnel $\mathrm{A}_{2}$ qui fournit cette tension $E_{r}$.

Ainsi, dans la bande passante de la boucle on a toujours $V_{x}=V_{1}$. L'appellation « charge asservie » est en fait impropre car la grandeur physique asservie n'est pas une charge mais la tension $V_{x}$, et la consigne de cet asservissement est une tension liée au courant $I_{x}$ par une impédance équivalente. Ce système est séduisant mais présente les mêmes inconvénients que le système d'injection asservi. Nous avons donc décidé d'exploiter le système de charge fictive réglable pour le nouvel équipement.

\subsection{Circuit à transimpédance}

Le circuit à transimpédance est constitué d'un transformateur de courant de métrologie (transformateur de courant à double circuit magnétique comme décrit dans fig. 10), dont le circuit primaire est inséré dans le circuit secondaire du transformateur à étalonner. Il fournit aux bornes d'un shunt inséré dans son circuit secondaire le signal proportionnel au courant $I_{x}$ servant à piloter les opérateurs vectoriels du dispositif de charge fictive et du dispositif d'injection de courant d'erreur. Les deux calibres de ce circuit (calibres 1 A et 5 A sur son circuit primaire) peuvent être commandés, soit manuellement, soit à partir du logiciel pendant les mesures automatiques.

Ce circuit est suivi d'un étage adaptateur d'impédance. Le gain de cet étage d'entrée est égal à 1,310 72 (65 536/50 000) pour que les pleines échelles soient obtenues avec des nombres programmés sur les convertisseurs numérique-analogique de 16 bits égaux à 50000 .

\section{Conception de la nouvelle électronique}

\subsection{Interconnexion de l'ensemble des éléments du nouveau système}

Les comparateurs de courants sont des dispositifs lourds et volumineux et les câbles ou barres portant le courant primaire sont susceptibles de rayonner des champs importants. Les éléments d'électronique sont donc nécessairement placés dans un coffret situé à distance, et le nombre de connexions entre celui-ci et les éléments extérieurs a été minimisé.

La figure 11 présente les interconnexions, en montrant les connexions à la terre métrologique. Le comparateur de courants et le transformateur à étalonner sont connectés à la terre, mais aucun enroulement ne l'est directement. C'est par le coffret de l'équipement électronique qu'ils peuvent l'être. Le boîtier du transformateur de mesure du courant secondaire est connecté à la terre par sa liaison de télécommande.

\subsection{Synoptique d'ensemble du nouveau système}

L'équipement électronique réalisé est contrôlable à distance par un ordinateur via une connexion USB, ce qui permet une automatisation complète : il n'y a aucune commande manuelle (commutateurs ou potentiomètres) car elles ont toutes été remplacées par des circuits électroniques. Cette modernisation a également pour conséquence une réduction importante du volume, l'ensemble est placé dans un coffret standard $(55 \times 56 \times 20) \mathrm{cm}^{3}$. Le synoptique de l'ensemble de cet équipement électronique est présenté en figure 12. Dans ce schéma :

- La tension image du courant secondaire $I_{x}$ entre sur l'amplificateur à haute impédance $\mathrm{A}_{1}$. Son gain est réglable pour pouvoir disposer de différents courants secondaires nominaux. Il est suivi par un amplificateur $A_{2}$ de gain -1 et d'un déphaseur $A_{3}$ lui-même suivi d'un autre amplificateur $\mathrm{A}_{4}$ de gain -1 . Ce déphaseur est chargé de fournir un signal en quadrature, et une commutation en fonction de la fréquence d'utilisation $(50 \mathrm{~Hz}, 60 \mathrm{~Hz}$ ou $400 \mathrm{~Hz}$ ). Suivent des commutateurs de signe et des convertisseurs numérique-analogique $\left(\mathrm{CNA}_{1}\right.$ à $\left.\mathrm{CNA}_{8}\right)$. Ils permettent de régler une fraction positive ou négative du signal entrant et du signal en quadrature. Les 


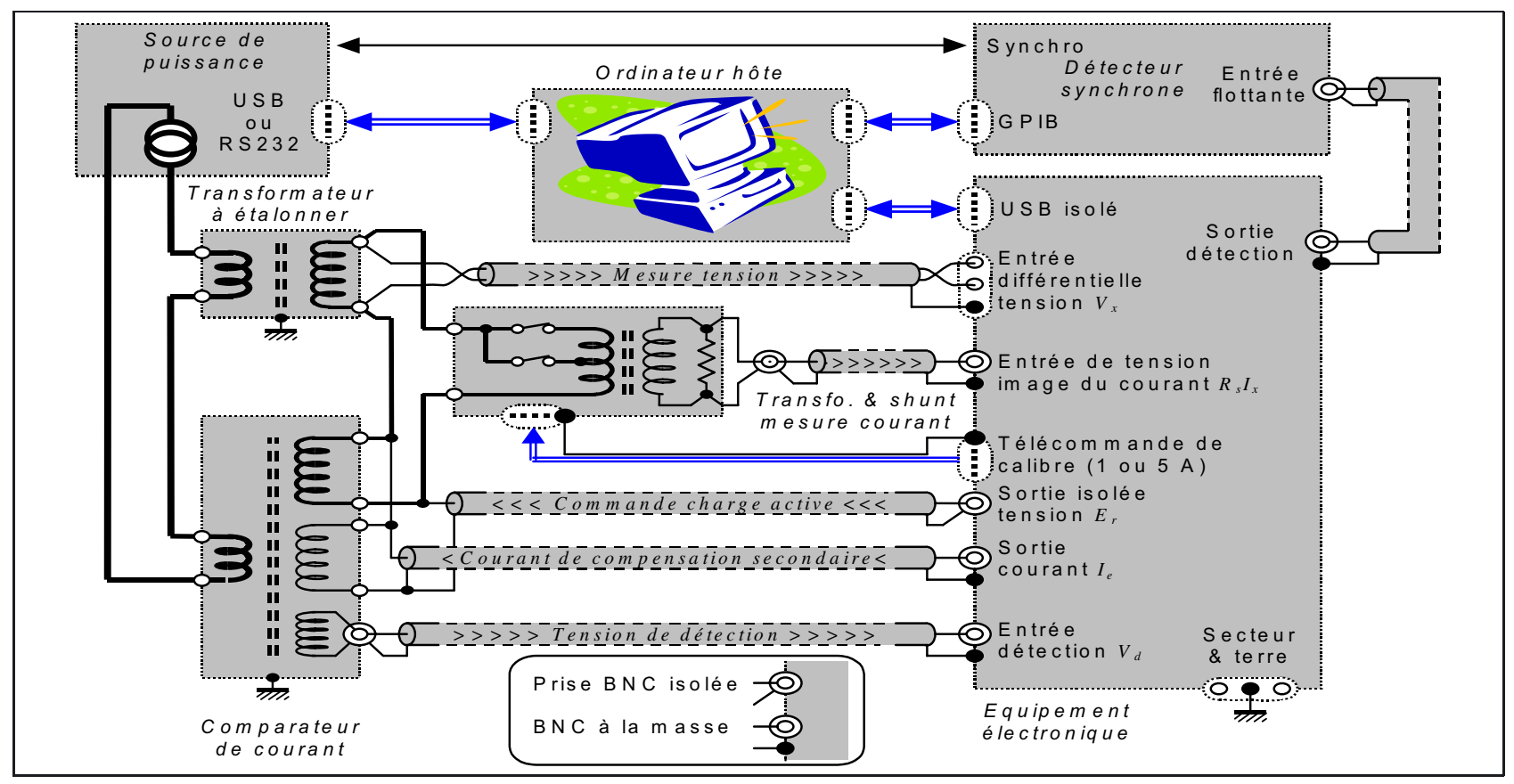

Fig. 11. - Interconnexions de l'ensemble des équipements avec le comparateur de courants et le transformateur en étalonnage.

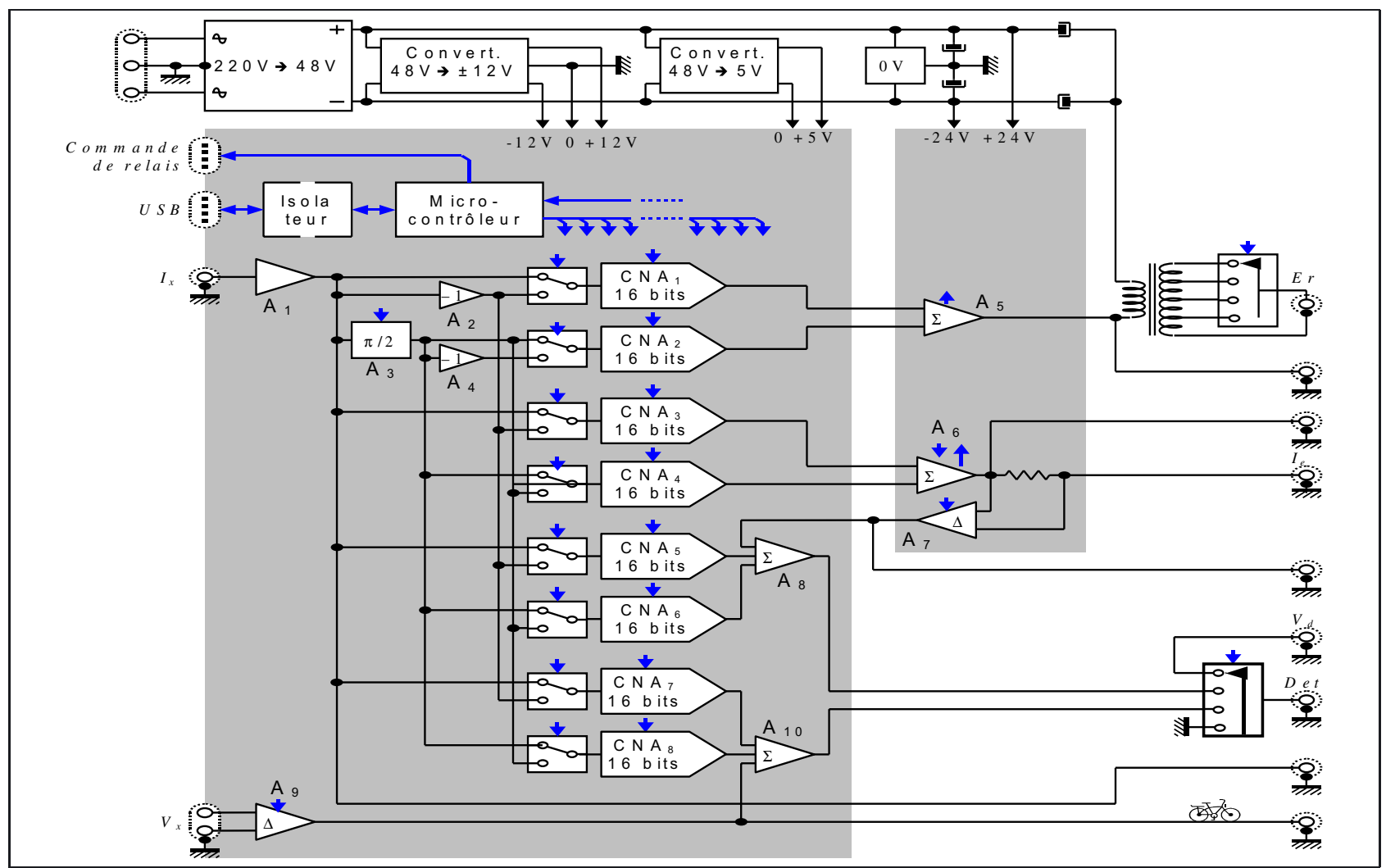

Fig. 12. - Schéma synoptique du dispositif électronique.

convertisseurs de rang impair et leurs commutateurs associés définissent ainsi des «parties réelles » et les autres des «parties imaginaires » de quatre vecteurs. Le courant d'erreur injecté dans l'enroulement de compensation secondaire est fourni par l'amplificateur $A_{6}$. Il s'agit d'un amplificateur opérationnel de puissance alimenté sur $\pm 24 \mathrm{~V}$, capable de fournir un courant important de l'ordre de $200 \mathrm{~mA}$ en crête (voir § 4.3.4). À son entrée, sont sommées les parties réelle et imaginaire du deuxième vecteur $\left(\mathrm{CNA}_{3}+\mathrm{CNA}_{4}\right)$. L'amplificateur comporte une commutation de gain pour disposer de plusieurs gammes de tension en sortie. En série dans cette sortie, une résistance permet de mesurer le courant par l'amplificateur différentiel $A_{7}$. 


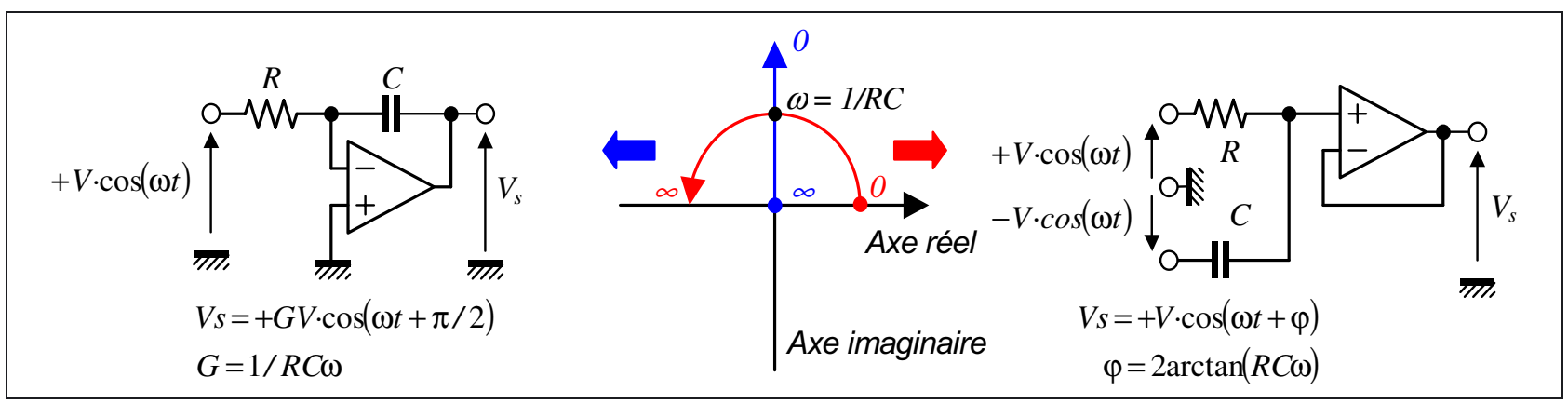

Fig. 13. - Intégrateur et filtre passe-tout.

Au signal sortant de celui-ci sont ajoutées dans le sommateur $\mathrm{A}_{8}$ les parties réelle et imaginaire du troisième vecteur $\left(\mathrm{CNA}_{5}+\mathrm{CNA}_{6}\right)$; le résultat qu'il faut annuler est mesuré par le détecteur synchrone branché sur la sortie « Det ». L'amplificateur $\mathrm{A}_{6}$ est doté d'un circuit de détection de saturation dont l'état peut être lu par le microcontrôleur. Il est également doté d'un circuit de contre-réaction particulier (fig. 16) qui assure une composante continue très faible (quelque $100 \mu \mathrm{V}$ au maximum).

- La tension de commande $E_{r}$ est fournie par un amplificateur opérationnel de puissance $\mathrm{A}_{5}$, au travers d'un transformateur dont on peut faire varier le rapport. En entrée, est effectuée la somme des parties réelle et imaginaire du premier vecteur $\left(\mathrm{CNA}_{1}+\mathrm{CNA}_{2}\right)$. L'amplificateur $\mathrm{A}_{5}$ (fig. 15) est lui aussi doté d'un circuit de détection de saturation. La tension $V_{x}$ apparaissant au secondaire du transformateur à étalonner est appliquée à l'entrée de l'amplificateur différentiel $\mathrm{A}_{9}$. Son schéma particulier (fig. 18) lui permet de supporter des tensions importantes. Son gain est lui aussi réglable pour disposer de plusieurs gammes de tensions. La sortie est sommée avec les parties réelle et imaginaire du quatrième vecteur $\left(\mathrm{CNA}_{7}+\mathrm{CNA}_{8}\right)$, le résultat qu'il faut annuler est lui aussi mesuré par le détecteur synchrone.

- L'ensemble des paramètres, gains, signes et composantes vectorielles sont télécommandables à distance au travers d'une liaison USB et d'un microcontrôleur. Le bus USB est isolé galvaniquement de la masse de l'équipement, il n'y a donc pas création d'une boucle de masse au travers de la masse de l'ordinateur hôte.

- Un module d'alimentation fournit une tension de $48 \mathrm{~V}$ avec une intensité maximale de $3 \mathrm{~A}$. Il alimente directement les deux amplificateurs de puissance. Un point milieu est nécessaire, qui est fourni par un pont diviseur suivi d'un amplificateur de gain unité repéré $0 \mathrm{~V}$ et de découplages adéquats. Dans ces découplages, circule le retour du courant alternatif fourni par $\mathrm{A}_{6}$, mais dans le circuit de masse fictive constituée par l'amplificateur $0 \mathrm{~V}$, il ne circule que très peu de courant continu : c'est pourquoi il n'est pas besoin d'une véritable alimentation double $\pm 24 \mathrm{~V}$. Un petit module convertisseur fournit la double alimentation $\pm 12 \mathrm{~V}$, utilisée par l'ensemble des circuits analogiques, et un autre fournit la tension $+5 \mathrm{~V}$ qui alimente le microcontrôleur et les quelques circuits logiques d'interface.

\section{3. Étude détaillée des composants}

\subsubsection{Le circuit déphaseur}

Le déphaseur, comme le montre la figure 13, peut être un intégrateur ou un filtre passe-tout. Les deux circuits utilisent exactement les mêmes composants : une résistance et un condensateur de précision, et un amplificateur opérationnel.

Avec des composants parfaits, l'intégrateur déphase toujours de $\pi / 2$. Mais l'amplitude est inversement proportionnelle à la fréquence. Le filtre passe-tout délivre une amplitude constante, mais c'est la phase qui tourne avec la fréquence. Dans les deux cas, il faut commuter la valeur d'au moins un des deux composants en fonction de la fréquence $(50 \mathrm{~Hz}, 60 \mathrm{~Hz}$ ou $400 \mathrm{~Hz})$, et s'accommoder de la médiocre précision du condensateur (1\%). Dans les deux cas, les pertes du condensateur ajoutent d'autres imperfections.

L'intégrateur présente l'inconvénient d'intégrer le courant continu. Même en l'absence de tension continue à l'entrée, un très faible courant de polarisation de l'amplificateur va le faire dériver jusqu'à saturation. Il faut ajouter une résistance ou un filtre passe-bas sur la contreréaction et on n'a plus alors un intégrateur parfait. Pour supprimer cet inconvénient, on peut réaliser un dérivateur en permutant la résistance et le condensateur sur le schéma de l'intégrateur. Mais c'est un circuit qui a théoriquement un gain infini à une fréquence infinie, c'est-àdire que le signal de sortie est très riche en composantes de bruit à haute fréquence.

Par ailleurs, au moment de l'application brutale d'un signal, les transitoires provoquent instantanément la saturation.

Ces considérations font que le filtre passe-tout est préférable. On peut réaliser des filtres passe-tout plus complexes ou d'ordre supérieur, avec des phases qui transitent plus ou moins brutalement. Ils nécessitent un plus grand nombre d'amplificateurs opérationnels, qui apportent leurs erreurs, et il vaut mieux s'en tenir à ce simple premier ordre. Le filtre de Hilbert qui déphase 
exactement de $90^{\circ}$ à toutes fréquences n'est qu'un opérateur mathématique physiquement irréalisable car non causal : son signal de sortie « commence» avant son signal d'entrée.

Le filtre passe-tout est un élément critique car sa stabilité et sa précision sont liées à celle du condensateur. Les résistances de fortes valeurs seraient des sources de bruit importantes et rendraient le système très sensible au courant de bruit en entrée de l'amplificateur qui suit. Il faut donc s'en tenir à quelques kilo-ohms pour les résistances; ce qui conduit, à $50 \mathrm{~Hz}$, à un condensateur de l'ordre du microfarad dont la plus grande précision qu'on puisse trouver n'est que $1 \%$. Nous avons donc, au voisinage de $R C \omega=1$,

$$
\varphi=2 \arctan (R C \omega) \Rightarrow \frac{\partial \varphi}{\partial C}=\frac{R \omega}{1+R^{2} C^{2} \omega^{2}} \Rightarrow \Delta \varphi \approx \frac{\Delta C}{2 C} .
$$

Cette erreur de $1 \%$ sur le condensateur se traduit donc par une erreur de phase de 0,005 rad, soit un rapport d'interaction de la composante supposée en quadrature sur le terme en phase égal à $\sin (0,005) \approx 5 \times 10^{-3}$, alors que l'erreur sur l'amplitude de la composante exactement en quadrature n'est que $1-\cos (0,005) \approx 1,25 \times 10^{-5}$. Pour faire mieux il convient donc de régler $R$ ou de refaire au minimum sur la composante en phase une composition vectorielle adéquate, c'est-à-dire y soustraire la projection de la composante pas tout à fait en quadrature. Cette composition peut être faite après calibrage soit par des moyens électroniques, soit par calculs dans le logiciel pilotant les mesures. C'est cette dernière méthode qui a été préférée car elle demande le moins de circuits électroniques et d'éventuelles mises au point.

Les autres termes d'erreur résultent des imperfections suivantes :

- la tolérance sur la résistance $R$. Avec une résistance à $0,001 \%$ et un coefficient de température de quelques $10^{-6} /{ }^{\circ} \mathrm{C}$, l'erreur est négligeable devant celle du condensateur. À $50 \mathrm{~Hz}$, la capacité parasite de la résistance (inférieure à $1 \mathrm{pF}$ ) a une influence négligeable;

- les pertes du condensateur. Elles se traduisent par un décalage de la phase et par une amplitude en sortie qui n'est plus égale à celle en entrée ;

- l'impédance de sortie des amplificateurs en amont. Un amplificateur opérationnel bouclé présente toujours une impédance de sortie qui comporte un terme résistif et un terme inductif. Les amplitudes et les phases en seront affectées, et pour maintenir une impédance très faible, un bon amplificateur à très large bande (quelques $\mathrm{MHz}$ ) et à très grand gain en boucle ouverte a été utilisé même si la fréquence maximale n'est que de $400 \mathrm{~Hz}$;

- l'impédance d'entrée de l'amplificateur de sortie et les capacités parasites en parallèle sur celle-ci ;

- la symétrie des deux tensions en entrée. Il y a nécessairement un amplificateur de gain -1 ou un

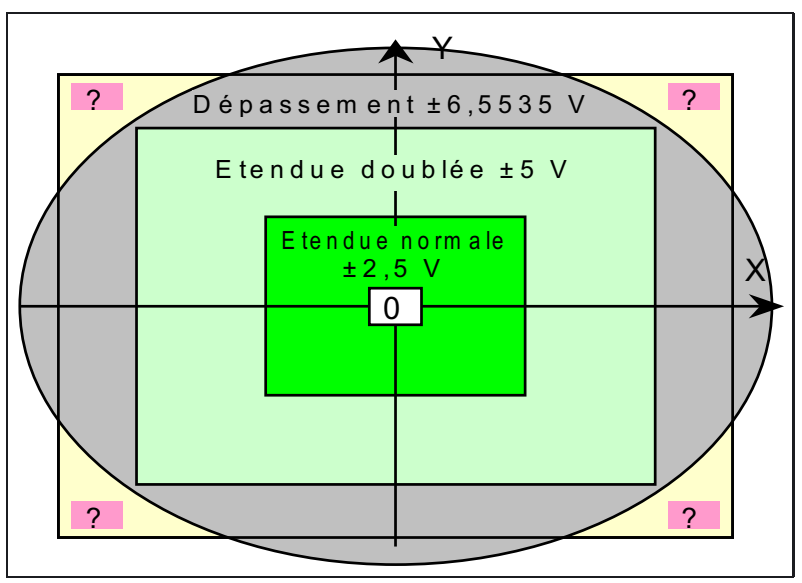

Fig. 14. - Étendues et dépassements.

transformateur : l'un comme l'autre apporte des erreurs de gain et de phase. Mais, là encore, on peut tenir facilement tenir $10^{-3}$;

- la commutation en fonction de la fréquence. Pour ne pas introduire de résistances gênantes, la commutation est réalisée par des relais électromécaniques bistables et non pas des semi-conducteurs (commutateurs CMOS).

\subsubsection{Convertisseurs numérique-analogique}

Les convertisseurs numérique-analogique utilisés ont une résolution de 16 bits, soit un nombre 0 à 65535 points. Pour que les résolutions soient des fractions simples des étendues, on a considéré que ces dernières sont exprimées pour 50000 points ; le complément jusqu'à 65535 étant un petit dépassement autorisé (soit un rapport 1,310 72). Ainsi la résolution est donc toujours $2 \times 10^{-5}$ fois l'étendue.

Ces étendues (fig. 14), s'étendent pour chacune des composantes réelles et imaginaires. Grâce aux deux enroulements primaires du transformateur de prélèvement et au gain 1,310 72 qui suit (fig. 10), on a toujours pour le courant secondaire nominal (5 A ou $1 \mathrm{~A}$ en fonction du calibre choisi sur le transformateur de prélèvement) 3,276 $8 \mathrm{~V}$ en entrée des opérateurs vectoriels c'est-àdire en entrée des convertisseurs numérique-analogique. En sortie de ceux-ci, pour cette étendue normale (gain = 50 000/65 536), la tension est exactement 2,5 V.

Lorsque le courant secondaire nominal est doublé, le module du vecteur composé peut dépasser la dynamique autorisée sur les amplificateurs qui suivent (en valeur crête $\pm 10 \mathrm{~V}$ à $\pm 11 \mathrm{~V}$ ); on ne doit pas atteindre les coins du carré (zones marquées «? » la fig. 14); en fait, les transformateurs à étalonner avec lesquels on pourrait avoir ce dépassement, sont en réalité très rares, voire inexistants.

Les erreurs de zéro, de gain et de phase peuvent être prises en compte avec un étalonnage, par contre la nonlinéarité des convertisseurs est difficile à extraire sans procédures très fastidieuses. 


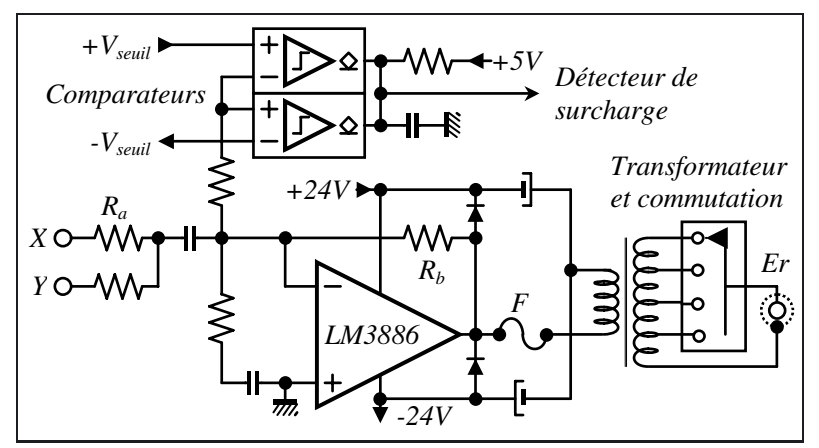

Fig. 15. - Amplificateur de puissance $\mathrm{A}_{5}$.

\subsubsection{Amplificateur de charge fictive}

L'amplificateur $\mathrm{A}_{5}$ (fig. 15) de la charge fictive fournit une tension $E_{r}$ réglée pour atteindre le rapport complexe $E_{x} / I_{x}$ désiré. L'usage normal du transformateur de courant à étalonner est bien de fournir de l'énergie à un shunt, énergie qui provient du primaire. Une partie de cette énergie est re-transférée au primaire par le comparateur de courants, et l'amplificateur ne va fournir ou absorber que la différence. La puissance apparente demandée à cette source, soit $E_{r} . I_{r}$ n'est qu' une petite fraction de celle demandée au transformateur mesuré, fraction qui dépend du comparateur de courants (page 88 de [3]). Pour réaliser cet amplificateur, nous avons utilisé le circuit intégré complet LM3886 qui peut dissiper jusqu'à $125 \mathrm{~W}$ sur un dissipateur de résistance thermique nulle, et fournir jusqu'à $60 \mathrm{~W}$ sur une résistance de $4 \Omega$.

Un transformateur de sortie à quatre calibres a été adjoint en sortie de cet amplificateur, couplé par des gros condensateurs placés en série dans le retour vers les alimentations. Nous avons ajouté deux diodes de protection utiles quand l'alimentation est hors tension. En parallèle sur l'entrée, un réseau RC série contribue à la stabilité de l'amplificateur en haute fréquence. En sortie, une thermistance CTP constitue un fusible réarmable F qui ajoute encore une protection contre les surintensités.

Les protections internes du LM3886 limitent le courant en sortie, et font donc que la tension n'est plus celle qui est définie par le rapport des résistances $R_{b} / R_{a}$, une tension apparaît alors sur son entrée négative. Un détecteur de surcharge qui consiste en deux comparateurs détecte la sortie de cette tension hors d'une plage comprise entre deux seuils d'environ $\pm 100 \mathrm{mV}$.

\subsubsection{Amplificateur de sortie du courant d'erreur}

Dans le cas le plus défavorable, c'est-à-dire, dans le cas d'un transformateur de courant à étalonner de forte erreur, par exemple étendue de mesure de $1 \%$, l'amplificateur $\mathrm{A}_{6}$ (fig. 16) doit pouvoir fournir un courant efficace allant jusqu'à $\sqrt{ } 2 \times 0,01$ fois le maximum du courant secondaire du transformateur à étalonner $(10 \mathrm{~A})$, soit en crête 0,2 A.

La tension de sortie et le gain nécessaire est variable avec l'étendue de mesure $(0,1 \%, 0,25 \%, 0,5 \%$

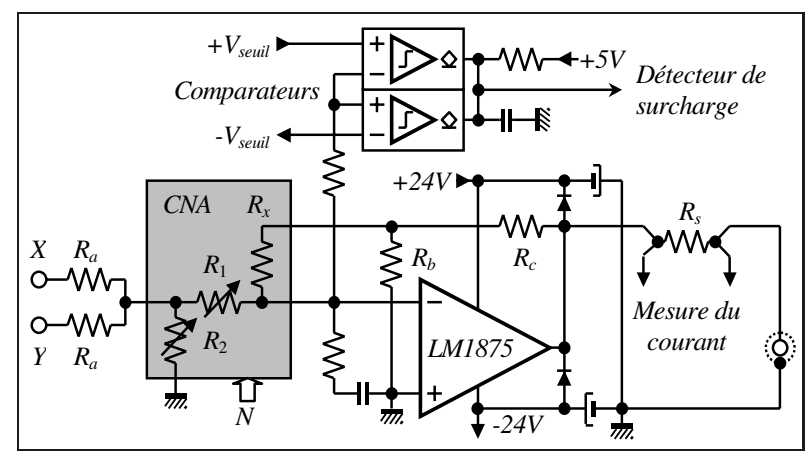

Fig. 16. - Amplificateur de puissance $\mathrm{A}_{6}$.

et $1 \%$ ), le courant secondaire nominal (1 A, 2 A, 5 A et $10 \mathrm{~A}$ ) et avec l'impédance de l'enroulement de compensation, elle-même dépendante des combinaisons des enroulements élémentaires placés en série ou en parallèle. Il n'est pas nécessaire que ce gain en tension soit précis, puisqu' on mesure par ailleurs le courant produit dans l'impédance du système qui est indéterminée. Pour s'accommoder de la plage de variation nécessaire tout en maintenant une résolution assez fine sur les deux composantes du vecteur d'action $\mathrm{CNA}_{3}$ et $\mathrm{CNA}_{4}$ (fig. 12), c'est un nouveau convertisseur numérique-analogique qui fixe le gain : il est utilisé comme un exposant commun aux mantisses programmées sur $\mathrm{CNA}_{3}$ et $\mathrm{CNA}_{4}$ car leur placement en cascade en multiplie les effets.

Ce convertisseur est équivalent à deux résistances $R_{1}$ et $R_{2}$ variables avec la commande numérique $N(0 \leq N \leq$ $65535)$ :

$$
\begin{aligned}
& R_{1}=65536 \times R_{x} / N \\
& R_{2}=65536 \times R_{x} /(65536-N)
\end{aligned}
$$

La valeur de $R_{x}$ est assez imprécise seuls comptent les rapports. Si $R_{b} / R_{c}=R_{a} / 2$, le gain global, proportionnel à $\mathrm{N}$ ne dépend pas de la valeur de $R_{x}$.

Avec $R_{a}=1,5 \mathrm{k} \Omega, R_{b}=1 \mathrm{k} \Omega$ et $R_{c}=3 \mathrm{k} \Omega$, le gain est ainsi égal à 4 pour $N=65536$. En utilisant les valeurs $N=2 P$ avec $0 \leq P \leq 15$, le gain est $1 / 16384 \leq G=$ $2(P-14) \leqslant 2$.

La résolution de 15 à 16 bits est donc assurée.

\subsubsection{Mesure du courant d'erreur injecté}

La combinaison des deux valeurs nominales du courant secondaire du transformateur à étalonner $(1 \mathrm{~A}$ ou $5 \mathrm{~A})$ avec celle des étendues de mesure $(0,05 \%$ à $1 \%)$ conduit à un rapport 50 sur l'étendue des courants d'erreur. Il faut faire varier dans ce rapport la résistance de mesure et le gain variable pour amener la tension lue sur la résistance série à la tension nominale $2,5 \mathrm{~V}$ (ou $5 \mathrm{~V}$ avec le courant doublé $2 \mathrm{~A}$ ou $10 \mathrm{~A}$ )

Pour ne pas faire appel à des gains importants, plus imprécis et plus courts en bande passante, le courant d'erreur injecté en sortie de l'amplificateur $\mathrm{A}_{6}$ est mesuré à travers deux résistances ( $25 \Omega$ pour un courant secondaire 


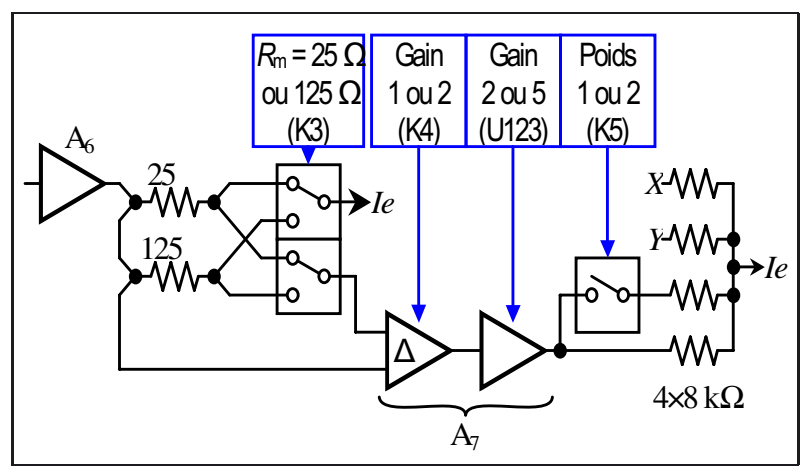

Fig. 17. - Mesure du courant injecté.

de $5 \mathrm{~A}$ ou $10 \mathrm{~A}$ et $125 \Omega$ pour un courant secondaire de 1 A ou 2 A). Un premier amplificateur différentiel de gain 1 ou 2 est suivi d'un autre amplificateur de gain 2 ou 5. En fonction de l'addition des deux composantes $X$ et $Y$ de l'opérateur vectoriel de mesure le poids 1 ou 2 est utilisé (fig. 17).

L'incertitude de la mesure est dépendante de celles de ces résistances de $25 \Omega$ et $125 \Omega$, et de celle du shunt de $10 \Omega$ du circuit à transimpédance (fig. 10).

\subsubsection{Mesure de la tension secondaire}

Les gammes de tensions différentielles aux bornes de l'enroulement secondaire du transformateur à étalonner qu'on peut atteindre dépendent du courant et de la charge demandée au transformateur mesuré. Le gain nécessaire est variable pour égaler cette tension à celle issue de l'opérateur vectoriel soit $2,5 \mathrm{~V}$ pour l'étendue nominale (50 000 points). L'impédance assignée aux bornes du transformateur à étalonner est $R+j X$, avec $-100 \Omega \leq$ $R \leq+100 \Omega$ et $-100 \Omega \leq X \leq+100 \Omega$. La puissance active est comprise entre $-100 \mathrm{~W}$ et $+100 \mathrm{~W}$, la puissance réactive entre $-100 \mathrm{~W}$ et $+100 \mathrm{~W}$, à $45^{\circ}$ la puissance apparente peut atteindre $100 \sqrt{ } 2 \mathrm{~V} \cdot \mathrm{A}$.

Les tensions d'entrée $V_{x}$ de l'amplificateur $\mathrm{A}_{9}$ peuvent être supérieures aux tensions d'alimentations, et son gain peut être inférieur à 1 ; ce qui ne peut être obtenu avec un montage à impédance d'entrée infinie. Cet amplificateur (fig. 18) est donc un montage différentiel classique à 4 résistances. Une commutation par relais permet de réaliser les gains 0,025 ou 0,5. La sortie de l'amplificateur est additionnée avec les deux composantes de l'opérateur vectoriel de mesure avec un poids commutable égal à 1 ou 4 : il en résulte les quatres valeurs de gain équivalent nécessaires.

\subsubsection{Le microcontrôleur}

Le système électronique est contrôlé par un microcontrôleur comprenant convertisseur analogiquenumérique à 12 bits. Son rôle est d'assurer :

- l'interface avec un ordinateur hôte par un bus USB ;

- la mesure numérique du courant secondaire ;

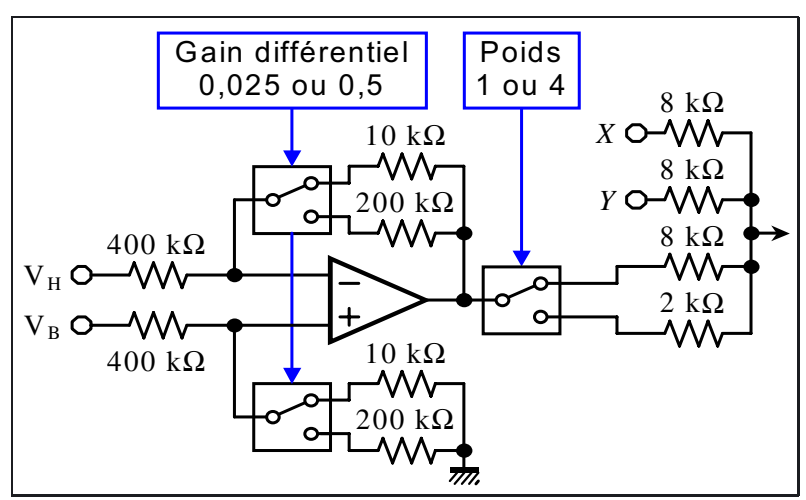

Fig. 18. - Mesure de tension secondaire $\left(\mathrm{A}_{9}\right)$.

- la commande des convertisseurs numériqueanalogique et des diverses commutations ;

- la surveillance des signaux de saturation des amplificateurs de puissance.

\section{Comportement des comparateurs de courants vis-à-vis des composantes harmoniques}

Les composantes harmoniques apparaissant aux bornes de l'enroulement de détection, $V_{d h}$, aux bornes de l'enroulement de compensation, $V_{c h}$, et aux bornes de l'enroulement secondaire du transformateur à étalonner $V_{x h}$. peuvent être écrites approximativement par les relations (8) et (9) :

$$
\begin{aligned}
& V_{c h}=-V_{x h} \approx-\frac{H_{x} Z_{s}}{\left(1-G \frac{N_{d}}{N_{c}}\right)}+\frac{H_{2} Z_{c 2}}{\left(1-\frac{N_{c}}{G N_{d}}\right)} \\
& V_{d h} \approx-\frac{N_{d} H_{x} Z_{s}}{\left(1-G \frac{N_{d}}{N_{c}}\right)}-\frac{N_{d} H_{2} Z_{c 2}}{N_{c}\left(1-G \frac{N_{d}}{N_{c}}\right)}
\end{aligned}
$$

avec :

$H_{x}$ : le courant harmonique fictif induit dans l'enroulement secondaire du transformateur à étalonner;

$H_{2}$ : le courant harmonique fictif induit dans l'enroulement secondaire du comparateur;

$Z_{c}$ : l'impédance de l'enroulement de compensation secondaire du comparateur;

$Z_{s}$ : l'impédance de sortie du dispositif d'injection ;

$Z_{c 2}$ : l'impédance de fuite de l'enroulement de compensation secondaire par rapport à l'enroulement secondaire du comparateur;

$N_{d}$ : le nombres de spires de l'enroulement de détection;

$N_{c}$ : le nombre de spires de l'enroulement de compensation secondaire;

$G$ : le gain du dispositif d'injection.

Il faut noter que l'analyse théorique des composantes harmoniques est faite avec l'hypothèse d'un courant primaire rigoureusement sinusoïdal. En fait, la teneur en 
harmoniques du courant primaire se traduit en principe par la même teneur en harmoniques de la tension aux bornes de l'enroulement secondaire du transformateur à étalonner, c'est-à-dire la même teneur en harmoniques que $V_{x h}$.

\section{Efficacité d'asservissement du dispositif électronique}

La question de l'efficacité de l'asservissement du dispositif d'injection, c'est-à-dire de l'approximation avec laquelle l'annulation de la composante fondamentale de la tension de détection, peut être exprimée en valeur relative de $I_{x}$ par la relation (10) :

$$
e f f=-\frac{E Z_{s}}{I_{x} Z_{c}\left(1-G \frac{N_{d}}{N_{c}}\right)}-\frac{E_{2} Z_{c 2}}{I_{x} Z_{c}\left(1-G \frac{N_{d}}{N_{c}}\right)}
$$

avec :

$I_{e}$ : le courant émis par le dispositif d'injection ;

$E$ : le courant d'erreur du transformateur à étalonner ;

$E_{2}$ : le courant magnétisant le comparateur;

$Z_{c}$ : l'impédance de l'enroulement de compensation secondaire du comparateur;

$Z_{S}:$ l'impédance de sortie du dispositif d'injection;

$\mathrm{Z}_{c 2}$ : l'impédance de fuite de l'enroulement de compensation secondaire par rapport à l'enroulement secondaire du comparateur;

$N_{d}$ : le nombres de spires de l'enroulement de détection ;

$N_{c}$ : le nombre de spires de l'enroulement de compensation secondaire;

$G$ : le gain du dispositif d'injection.

L'application de la relation (10) n'est valable qu'aux seules composantes fondamentales des courants. Le premier terme est généralement très inférieur au deuxième terme. On constate que l'efficacité d'asservissement dépend essentiellement de $Z_{s}$ d'une part et du couplage du comparateur dont dépend $Z_{c}, Z_{c 2}, N_{d}$ et $N_{c}$.

\section{Incertitude d'étalonnage d'un transformateur de courant}

Les composantes d'incertitude sont réparties en deux groupes : celles liées au comparateur de courant et celles liées au dispositif électronique de mesure.

\subsection{Composantes d'incertitude liées au comparateur de courants}

\subsubsection{Erreurs capacitive et magnétique}

On peut distinguer deux sources d'erreur : erreur magnétique et erreur capacitive [6]. La procédure pour évaluer et éliminer ces erreurs est détaillée dans [1]. Les deux comparateurs de courants du LNE ont des erreurs magnétique et capacitive nulles. Elles ont été évaluées avec des incertitudes meilleures que $1 \mu \mathrm{A} / \mathrm{A}$ pour l'erreur de rapport et meilleures que $1 \mu \mathrm{rad}$ pour le déphasage.

\subsubsection{Influence de la température}

Les comparateurs de courants sont généralement peu sensibles à la température. Les mesures réalisées dans un environnement non régulé en température ont montré que l'influence de la température est difficilement détectable par une droite de moindres carrés. Pour les comparateurs du LNE, cette influence est meilleure que $0,3 \mu \mathrm{A} /\left(\mathrm{A} \cdot{ }^{\circ} \mathrm{C}\right)$ pour l'erreur de rapport et meilleure que $0,3 \mu \mathrm{rad} /{ }^{\circ} \mathrm{C}$ pour le déphasage.

\subsubsection{Auto-échauffement}

Pour évaluer l'auto-échauffement des comparateurs, deux mesures peuvent être réalisées : une mesure à $0,05 \times I_{n}\left(I_{n}\right.$ est le courant nominal) pendant la montée en courant et, après avoir maintenu le courant pendant plusieurs minutes à $1,2 \times I_{n}$, une deuxième mesure est refaite immédiatement à $0,05 \times I_{n}$. L'écart entre les deux mesures donne une estimation de l'influence de l'autoéchauffement. Pour les comparateurs du LNE cette influence est meilleure que $0,5 \mu \mathrm{A} / \mathrm{A}$ pour l'erreur de rapport et meilleure que 0,5 rad pour le déphasage.

\subsection{4. État magnétique des comparateurs}

Les comparateurs de courants se magnétisent assez facilement, cette magnétisation peut apparaître au cours des mesures. Pour évaluer cette influence deux mesures sont réalisées ; une mesure quand le comparateur est totalement démagnétisé et une mesure après une forte magnétisation volontaire. L'écart entre les deux mesures donne une estimation de l'influence de l'état magnétique du comparateur. Pour les comparateurs du LNE cette influence est meilleure que $0,3 \mu \mathrm{A} / \mathrm{A}$ pour l'erreur de rapport et meilleure que $0,3 \mu \mathrm{rad}$ pour le déphasage.

\subsubsection{Circuit primaire}

La position du conducteur de courant primaire dans l'ouverture centrale du comparateur peut avoir une influence non négligeable. Cette influence peut être décelée en réalisant des mesures tout en faisant des translations horizontales et verticales autour de la position centrale de l'ouverture centrale du comparateur. Pour les comparateurs du LNE, cette influence est estimée à 2,5 $\mu \mathrm{A} / \mathrm{A}$ pour l'erreur de rapport et à $1,5 \mu$ rad pour le déphasage. Pour la réduire à $1 \mu \mathrm{A} / \mathrm{A}$ pour l'erreur de rapport et à $1 \mu \mathrm{rad}$ pour le déphasage, il a été décidé de choisir une position de référence (position centrale). 
7.2. Composantes d'incertitude liées au dispositif électronique de mesure

\subsubsection{Elaboration du signal de référence}

Cette composante proportionnelle à $I_{x}$ provient pendant l'élaboration du signal de référence. Elle prend en compte les erreurs des amplificateurs de gain -1 , le circuit à transimpédance et le déphaseur. À l'aide d'un étalonnage cette influence peut être inférieure à $0,1 \mu \mathrm{A} / \mathrm{A}$ pour l'erreur de rapport et inférieure à $0,1 \mu \mathrm{rad}$ pour le déphasage.

\subsubsection{Mesure du courant d'erreur}

Cette composante proportionnelle à $I_{x}$ provient principalement, dans le circuit de mesure, du courant d'erreur $I_{e}$ (la résolution et la linéarité des convertisseurs $\mathrm{CNA}_{5}$ et $\mathrm{CNA}_{6}$, le gain des amplificateurs $\mathrm{A}_{7}$ et $\mathrm{A}_{8}$, le rapport entre la résistance de mesure et la résistance implantée dans le circuit à transimpédance). À l'aide d'un étalonnage, cette influence peut être inférieure à $0,3 \mu \mathrm{A} / \mathrm{A}$ pour l'erreur de rapport et inférieure à $0,3 \mu \mathrm{rad}$ pour le déphasage.

\subsubsection{Mesure de la tension secondaire}

Cette composante proportionnelle à $I_{x}$ provient principalement dans la mesure, de la tension secondaire $V_{x}$ (la résolution et la linéarité des convertisseurs $\mathrm{CNA}_{7}$ et $\mathrm{CNA}_{8}$, le gain des amplificateurs $\mathrm{A}_{10}$ et $\mathrm{A}_{9}$ et l'influence des résistances d'entrée de l'amplificateur $A_{9}$ ). À l'aide d'un étalonnage cette influence peut être inférieure à $0,1 \mu \mathrm{A} / \mathrm{A}$ pour l'erreur de rapport et inférieure à $0,1 \mu \mathrm{rad}$ pour le déphasage.

\subsubsection{Tension résiduelle aux bornes de l'enroulement de détection}

Le résidu de tension $V_{d}$ (harmoniques inclus) aux bornes de l'enroulement de détection mesurée avec le détecteur synchrone est proportionnel à l'efficacité d'asservissement du dispositif d'injection de courant d'erreur $I_{e}$. L'efficacité est estimée pendant chaque étalonnage en divisant la tension résiduelle $V_{d}$ par la sensibilité du comparateur, de l'ordre de $4 \mathrm{~V}$ par ampère tours, le nombre de spires de l'enroulement secondaire du comparateur et par le courant secondaire.

\subsubsection{Non linéarité de la charge}

Cette composante provenant du résidu de tension mesuré avec le détecteur synchrone pendant l'opération de mesure de la tension $V_{x}$ (détecteur synchrone sur la sortie de l'amplificateur $\mathrm{A}_{10}$ ) dépend fortement de l'influence de la charge sur le transformateur en étalonnage. Par exemple si cette tension est égale à $20 \mathrm{mV}$ (harmoniques inclus) pour une charge secondaire de $10 \mathrm{~V} \cdot \mathrm{A}(\cos \varphi=1)$ et pour $I_{x}=5 \mathrm{~A}$ donc $V_{x}=2 \mathrm{~V}$, l'erreur est égale à $20 \mathrm{mV} / 2 \mathrm{~V}$, ce qui donne une erreur sur la charge de

\section{Tableau 1}

Bilan d'incertitude pour l'étalonnage d'un transformateur de courant de forte intensité à l'aide de comparateurs de courants.

\begin{tabular}{|c|c|c|}
\hline \multirow[b]{2}{*}{ Sources d'incertitudes } & \multicolumn{2}{|c|}{ Incertitude type $(k=1)$} \\
\hline & $\begin{array}{c}\text { Erreur de } \\
\text { rapport } \\
(\boldsymbol{\mu} \mathrm{A} / \mathrm{A})\end{array}$ & 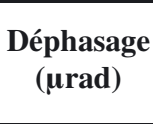 \\
\hline \multicolumn{3}{|c|}{ Composantes liées au comparateur } \\
\hline Erreur capacitive et magnétique & 1 & 1 \\
\hline $\begin{array}{l}\text { Influence de la température } \\
\left(\Delta T=2{ }^{\circ} \mathrm{C}\right)\end{array}$ & 0,6 & 0,6 \\
\hline Auto-échauffement & 0,5 & 0,5 \\
\hline État magnétique & 0,3 & 0,3 \\
\hline Circuit primaire & 1 & 1 \\
\hline \multicolumn{3}{|c|}{ Composantes liées au dispositif électronique } \\
\hline $\begin{array}{l}\text { Elaboration du signal de } \\
\text { référence }\end{array}$ & 0,1 & 0,1 \\
\hline Mesure du courant d'erreur & 0,3 & 0,3 \\
\hline Mesure de la tension secondaire & 0,1 & 0,1 \\
\hline Efficacité d'asservissement & 1 & 1 \\
\hline $\begin{array}{l}\text { Non-linéarité de la charge } \\
(0,05 \mathrm{~V} \cdot \mathrm{A})\end{array}$ & 2 & 2 \\
\hline $\begin{array}{l}\text { Sensibilité du détecteur } \\
\text { synchrone }\end{array}$ & 1 & 1 \\
\hline Incertitude élargie $(k=2)$ & 6 & 6 \\
\hline
\end{tabular}

$0,1 \mathrm{~V} \cdot \mathrm{A}$. Si le transformateur de courant à une variation de $50 \mu \mathrm{A} /(\mathrm{A} \cdot \mathrm{V} \cdot \mathrm{A})$ et $25 \mu \mathrm{rad} / \mathrm{V} \cdot \mathrm{A}$, cette composante est alors égale à $5 \mu \mathrm{A} / \mathrm{A}$ pour l'erreur de rapport et égale à 2,5 $\mu \mathrm{rad}$ pour le déphasage.

\subsubsection{Sensibilité du détecteur synchrone}

Le détecteur synchrone intervient plusieurs fois dans l'étape de la mesure. Sa sensibilité n'est importante que lorsqu'il est connecté en sortie de l'amplificateur $A_{8}$. Elle est inversement proportionnelle à l'amplitude du courant d'essais. Elle reste inférieure à $1 \mu \mathrm{A} / \mathrm{A}$ pour l'erreur de rapport et inférieure à $1 \mu \mathrm{rad}$ pour le déphasage entre le courant primaire et le courant secondaire.

\subsubsection{Perturbations sur le réseau électrique}

La phase du réseau électrique peut affecter les mesures lorsque le courant d'essais ne dépasse pas $0,05 \times I_{n}$. Pour s'en affranchir, il est préférable de faire deux mesures en permutant le sens du courant d'essais et d'utiliser deux boucles de courant symétriques.

\subsection{Bilan d'incertitude}

En conclusion le bilan d'incertitude d'étalonnage d'un transformateur de courant est synthétisé dans le tableau 1. Il ne tient pas compte des incertitudes liées au transformateur à étalonner. 


\section{Conclusion}

Les deux comparateurs de courants conçu au LCIE présentent d'excellentes caractéristiques; les erreurs magnétique et capacitive sont maitrisées et rendues inférieures à $1 \mu \mathrm{A} / \mathrm{A}$ pour l'erreur de rapport et à $1 \mu \mathrm{rad}$ pour le déphasage. Les dispositifs électroniques qui ont été conçus pour faciliter l'emploi du comparateur ne parvenaient pas à exploiter pleinement sa performance métrologique. L'efficacité et la stabilité d'asservissement dépendaient fortement de couplages du comparateur et du transformateur à étalonner. Il était très difficile de réaliser un asservissement inconditionnellement stable.

Le LNE a modernisé les deux dispositifs associés aux comparateurs de courants, à savoir, le dispositif de charge fictive et le dispositif d'injection de courant d'erreur. Le système à asservissement direct exploité dans les anciens équipements a été abandonné et remplacé par un asservissement par logiciel à lecture directe.

Ces nouveaux dispositifs permettront, d'une part, d'exploiter pleinement la performance métrologique des comparateurs; on pourra avoir un asservissement stable et efficace quelque soit les couplages du comparateur et

Article reçu le 18 novembre 2013, version révisée reçue le 15 septembre 2014. du transformateur à étalonner et ainsi garantir, dans tous les cas, une faible incertitude de quelques $\mu \mathrm{A} / \mathrm{A}$ pour l'erreur de rapport et de quelques urad pour le déphasage et, d'autre part, d'automatiser complètement les mesures.

\section{Références}

[1] KNosP R., «L'emploi de comparateurs de courants alternatifs dans la métrologie des courants de forte intensité », RGE, 1981, 350-357.

[2] Kusters N.L. et Moore W.M., "The compensated current comparator, A new reference standard for current transformer calibration in industry", IEEE, 1964, 107114.

[3] Silsbee F.B., SMith R.L., Forman N.L. et PARK. J.H., "Equipment for testing current transformers", Part of bureau of standard journal of research, 11, 1933.

[4] Kusters N.L. et Moore W.J., "A phantom burden for current transformer calibration”, IEEE, 1974, 240-243.

[5] Kusters N.L. et Moore W.J., "Phantom burden arrangement for current transformer calibration. Canadian patents and development limited", Ottawa, Canada, aout 1971.

[6] Kusters N.L. et Moore W.J., "Capacitive error in current comparators", IEEE, 1964, 210-216. 